\title{
Broad spectrum insect resistance and metabolites in close relatives of the cultivated tomato
}

\author{
Ben Vosman • Wendy P. C. van't Westende - Betty Henken - Henriëtte D. L. M. van Eekelen • \\ Ric C. H. de Vos $\cdot$ Roeland E. Voorrips
}

Received: 30 June 2017 / Accepted: 29 January 2018/Published online: 6 February 2018

(C) The Author(s) 2018. This article is an open access publication

\begin{abstract}
Wild relatives of tomato possess effective means to deal with several pests, among which are a variety of insects. Here we studied the presence of resistance components against Trialeurodes vaporariorum, Myzus persicae, Frankliniella occidentalis, and Spodoptera exigua in the Lycopersicon group of Solanum section Lycopersicon by means of bioassays and comprehensive metabolite profiling. Broad spectrum resistance was found in Solanum galapagense and a few accessions of $S$. pimpinellifolium. Resistance to the sap sucking insects may be based on the same mechanism, but different from the caterpillar resistance. Large and highly significant differences in the leaf metabolomes were found between $S$. galapagense, containing type IV trichomes, and its closest relative $S$. cheesmaniae, which lacks type IV trichomes. The most evident differences were the relatively high levels of different methylated forms
\end{abstract}

Electronic supplementary material The online version of this article (https://doi.org/10.1007/s10681-018-2124-4) contains supplementary material, which is available to authorized users.

B. Vosman $(\varangle)$. W. P. C. van't Westende .

B. Henken · R. E. Voorrips

Plant Breeding, Wageningen University and Research, P.O. Box 386, 6700 AJ Wageningen, The Netherlands e-mail: ben.vosman@wur.nl

H. D. L. M. van Eekelen - R. C. H. de Vos Bioscience, Wageningen University and Research, P.O. Box 16, 6700 AA Wageningen, The Netherlands of the flavonoid myricetin and many acyl sucrose structures in $S$. galapagense. Possible candidate genes regulating the production of these compounds were identified in the $W f-1$ QTL region of $S$. galapagense, which was previously shown to confer resistance to the whitefly $B$. tabaci. The broad spectrum insect resistance identified in $S$. galapagense will be very useful to increase resistance in cultivated tomato.

Keywords Lycopersicon group · Whitefly · Aphid · Thrips · Caterpillar · Metabolomics · Acyl sugars · Flavonoids

\section{Introduction}

Plants have evolved all kinds of adaptations to survive insect attacks. Their defense mechanisms interfere with the physiology and/or behaviour of the insect and may include among others the presence of (glandular) trichomes, wax layers, defensive metabolites or proteins. They are coined together as direct defense when resistance components of the plant directly interact with the attacker. As plants are facing not just one but a whole suite of attackers, they evolved a variety of mechanisms to deal with insects. Resistance mechanisms may be highly attacker-specific and interact with only one biotype of an insect, or they may be more general and protect the plant from a range of 
attackers (Agrawal 2011; Broekgaarden et al. 2011; Smith and Boyko 2007; Thompson 2005).

Cultivated tomato (Solanum lycopersicum) suffers from a wide range of arthropods, mainly generalists representing different feeding guilds. They cause direct damage through feeding, but often the greatest harm is done by the viruses they transmit. Resistance against insects and mites is found in several wild relatives of the cultivated tomato. Well known sources are Solanum pennellii and S. habrochaites (Muigai et al. 2003). Recently also some more closely related wild relatives of the cultivated tomato have been added to the list, including $S$. pimpinellifolium and $S$. galapagense showing resistance to the whitefly Bemisia tabaci (Firdaus et al. 2012; Lucatti et al. 2013; Rodriguez-Lopez et al. 2011), and the twospotted spider mite, Tetranychus urticae (Alba et al. 2009; Rakha et al. 2017a). Currently it is unknown if also other insect species are affected by the resistance present in the Lycopersicon group of Solanum section Lycopersicon and if such resistance in the different members is governed by the same mechanism.

Solanum pimpinellifolium and $S$. galapagense are part of the Lycopersicon group of Solanum section Lycopersicon that also includes $S$. cheesmaniae and the cultivated tomato (Peralta and Spooner 2005; Peralta et al. 2008). Especially $S$. cheesmaniae and $S$. galapagense are genetically very close (Lucatti et al. 2013) and both species are endemic to the Galapagos islands. However, they are easily distinguishable based on leaf morphology (Darwin et al. 2003). They also differ strongly in the presence of type IV trichomes, which are present on S. galapagense and absent on S. cheesmaniae leaves (Lucatti et al. 2013). Type IV trichomes are also found on some accessions of S. pimpinellifolium, but not on S. lycopersicum (Firdaus et al. 2012). The presence/absence of type IV trichomes has been genetically mapped in an F2 population derived from a $S$. lycopersicum $\times S$. galapagense cross and a major Quantitative Trait Locus (QTL) $W f-1$ was identified on the end of chromosome 2 (Firdaus et al. 2013).

In Solanum section Lycopersicon insect and mite resistance has been suggested to be based on specific plant compounds present in the glandular trichomes, including acyl sugars, terpenes, methyl ketones and flavonoids (Glas et al. 2012). Acyl sugars have received a lot of attention and several genes involved in the production and modification of acyl sugars have been identified (Fan et al. 2016; Schilmiller et al. 2012, 2015, 2016). Acyl sugars may be directly toxic to the insects (Luu et al. 2017), but it is also possible that their sticky nature causes death of the insect by immobilizing them on the leaf (Schilmiller et al. 2008; Rakha et al. 2017a). There are many different acyl sugars produced in a tomato plant and its wild relatives (Lucatti et al. 2013; McDowell et al. 2011), possibly exerting different effects on different insect species. However, apart from acyl sugars other compounds present in the glandular trichomes may be key to insect resistance as well. Firdaus et al. (2013) analyzed the acyl sugar content in bulks of the 10 most resistant and 10 most susceptible plants of the F2 population derived from the $S$. lycopersicum $\times S$. galapagense cross and found large differences in the abundance $(>100 \times$ difference); high abundance in resistant plants, low abundance in susceptible plants. This strongly suggests that the $W f-l$ QTL is also involved in acyl sugar production, although other QTLs may play a role as well.

In the current study we aimed to obtain basic information on the extent of insect resistance identified in the Lycopersicon group. More specifically we investigated (1) whether there is, next to $B$. tabaci resistance, also resistance against the phloem-feeding Trialeurodes vaporariorum and Myzus persicae, the cell content-feeding thrips Frankliniella occidentalis, and to the leaf feeding caterpillar Spodoptera exigua, (2) whether the different accessions are equally effective against the different insects, and (3) which metabolites present in the leaves, as detected by using comprehensive Liquid Chromatography Mass Spectrometry (LCMS) metabolite profiling, of the different members of the Lycopersicon group correlate to resistance characteristics.

\section{Materials and methods}

Plant materials and growing conditions

Based on genetic relationship, resistance to B. tabaci, presence/absence of trichome type IV and acyl sugar profiles which we had available for approx. 100 accessions of the Lycopersicon group of Solanum section Lycopersicon, we selected 5 accessions of $S$. pimpinellifolium, 3 of $S$. cheesmaniae and 3 of $S$. galapagense for the current study to cover the 
variation within the group and complemented this with 4 reference accessions; see Supplementary Table 1 for details on the materials. The seeds were sown and 2 weeks later the young plants were transferred to $17 \mathrm{~cm}$ pots with potting compost and grown in a greenhouse at $23 / 19{ }^{\circ} \mathrm{C}$ (day/night temp), relative humidity (RH) $70 \%$, light 16/8 h (day/night) at Unifarm, Wageningen, The Netherlands. Plants were randomized in blocks with one plant per block. The insect resistance evaluations started when the plants were approx. Six weeks old. Five plants per accession were used in the tests for whitefly, thrips and caterpillar resistance tests, and 7 plants per accession for the aphids resistance test.

Insect rearings and resistance evaluations

A rearing of the whitefly Trialeurodes vaporariorum was obtained from Wageningen University and Research Greenhouse Horticulture, Bleiswijk, NL and maintained on tomato cv. Moneymaker. Synchronized 1 day old adult whiteflies were used for the experiment. Each plant received 3 clip-on cages containing 5 female whiteflies. The clip-on cages were placed on the abaxial side of the first, second and occasionally the third fully expanded leaves, in which numbering starts from the top of the plant, the first fully expanded leaf being the youngest. After 5 days the number of living and dead whiteflies and the number of eggs were counted.

The thrips Frankliniella occidentalis was obtained from the same origin and reared on 2 different hosts [common bean (Phaseolus vulgaris) and tomato cv. Moneymaker]. Small leaflets (5 pieces) from the first or second fully expanded leaf of each plant were inserted with their petiole in a drop $1.5 \%$ water agar that was present in a Petri dish (Falcon Tight-Fit Lid Dish $50 \times 9 \mathrm{~mm}$ style) to prevent drying out. Five adult female thrips of assorted age were placed on the leaflet and survival of the thrips was observed at day 2 , 3 and 4 after the start of the infestation. There were no significant differences observed between thrips reared on common bean versus those reared on tomato, based on the analysis of 3 plant that received bean-reared thrips and 2 plants that received tomato-reared thrips per accession (Anova thrips origin: $\mathrm{p}=0.974$ ).

Eggs from the caterpillar Spodoptera exigua were obtained from Entocare C.V. (Wageningen, NL). Larvae were reared on tomato (cv. Moneymaker) and allowed to pupate in perlite. Adult moths were fed with $10 \%$ sucrose solution and allowed to deposit eggs on paper. Eggs were put at $25^{\circ} \mathrm{C}$ and after hatching the one-day old larvae were used for the experiments. From each plant a small leaflet from the first, second and third fully expanded leaves was taken and inserted with its petiole in a drop of $1.5 \%$ water agar that was present in a Petri dish or a small container to prevent drying out. Five larvae were placed on a leaflet. When a leaflet was eaten for more than 50\%, a new leaflet from the same plant was added to the Petri dish/container. The experiment was carried out in a growing chamber at $25{ }^{\circ} \mathrm{C}$ and $65 \% \mathrm{RH}, 16 \mathrm{~h}$ day $/ 8 \mathrm{~h}$ night. Larval survival and weight was measured 15 days after the start of the infestation.

A rearing of the aphid Myzus persicae was maintained on tomato cv. Moneymaker. Synchronized oneday old nymphs were used for the experiment. Each plant obtained 2 clip-on cages containing 5 nymphs. The clip-on cages were placed on the abaxial side of the first and second fully expanded leaves. After 12 days the number of living aphids and next generation nymphs were counted.

\section{LCMS profiling of leaves}

With this experiment we aimed to measure the "chemical environment" of the plant leaves as experienced by the insects. However, the leaf tissue that was in direct contact with the insect, i.e. under the clip cage is not representative after an infestation (due to e.g. waxes, feces, honeydew, mold and bacterial growth). The alternative to take a sample directly outside the clip cage was also difficult as especially the $S$. galapagense type accessions have very small leaves. Therefore, we choose to sample the leaf opposite of that containing the clip cage. By using these leaves, potential differences in the leaf metabolomes due to differential growth conditions and plant development were excluded as much as possible. Leaves were harvested after finishing the bioassays, to prevent possible effects on the resistance assay. However, in this case a systemic response resulting from the aphid infestation of the opposite leaf may have occurred.

Leaves were frozen in liquid nitrogen and stored at - $80{ }^{\circ} \mathrm{C}$ until use. Each sample for metabolomics analysis contained equal amounts of leaf tissue from two plants. Frozen leaves were ground into a fine 
powder and $100 \mathrm{mg}$ fresh weight of leaf powder was weighed for metabolite extraction with $300 \mu \mathrm{l} 75 \%$ $\mathrm{MeOH}$ containing $0.1 \%$ formic acid (FA). Five technical replicates from a pool of samples were similarly prepared and used as so-called quality control samples (QCs) to estimate overall analytical variability for each metabolite detected (De Vos et al. 2007). LC-MS was performed on a Waters Acquity HPLC coupled to a Thermo Ion Trap-Orbitrap FTMS hybrid MS system, using a Phenomenex Luna C18 (2) column and a gradient of water and acetonitrile, both acidified with $0.1 \%$ FA, as described by (Firdaus et al. 2013). The Orbitrap FTMS was operating in negative ionization mode at a mass resolution of 60,000 (FWHM) and a mass range of m/z 90-1350 D., details in (van der Hooft et al. 2011). Metalign software (Lommen 2009) was used for unbiased mass peak picking. Extracted mass peaks corresponding to acyl sugars were annotated based on the specific accurate mass of their formic acid adduct $[\mathrm{M}+\mathrm{HCOOH}-\mathrm{H}]^{-}$, which was calculated from the elemental formula, in combination with their specific retention times (Firdaus et al. 2013), using the Search_LC option of Metalign (Lommen et al. 2011) with a maximal mass deviation of $5 \mathrm{ppm}$ and a retention time window of $0.1 \mathrm{~min}$. Acyl sugars were coded according to the sugar backbone ( $\mathrm{G}$ for glucose and $\mathrm{S}$ for sucrose), number of acyl chains and total number of carbon atoms in these acyl chains. For instance, S3:20 refers to sucrose acylated with 3 chains with in total 20 carbon atoms. For a number of acyl sugars, additional MSMS (= MS2) and MS3 fragmentation experiments provided information on the distribution of the individual acyl chains, e.g. S3:20 (5.5.10) refers to sucrose with acyl chains of 5,5 and 10 carbons, respectively. Subsequently, all 114,807 mass signals extracted were aligned across all samples in an untargeted manner and the resulting peak list was further processed using MSClust (Tikunov et al. 2012), in order to group redundant mass signals originating from the same molecule, including fragments, adducts and their isotopes. The resulting list with the relative abundances (based on peak heights) of each metabolite in each sample was used for statistical analyses. Selected metabolites most significantly correlating with insect resistance were manually annotated from the observed accurate masses of the molecular ion and its fragments, as well as absorbance spectra recorded by the in-line photodiode array detector using both publicly accessible and in-house LCMS metabolite databases from previous studies on tomato fruits (Moco et al. 2007) and seedlings (Roldan et al. 2014).

\section{Data analysis}

Per plant the observations from the Petri dishes/containers or clip-on cages were combined.

Whitefly data Survival was expressed as (living whiteflies)/(living + dead whiteflies); the total number of whiteflies could be less than 15 as some escaped from the cages; cages with less than 4 whiteflies and plants with less than 2 remaining cages were discarded. The numbers of eggs were divided by the estimated average number of living whiteflies present, calculated as (2*living whiteflies + dead whiteflies)/ 2. For ANOVA analysis these data were transformed to obtain a more or less constant residual variance: survival as arcsine[sqrt(x)] and eggs as $\operatorname{sqrt}(\mathrm{x})$.

Thrips data Survival was expressed as (living thrips)/(living + dead thrips). For ANOVA analysis these data were transformed as arcsine[sqrt(x)].

Caterpillar data When a caterpillar could not be found back (probably completely dried out), it was counted as dead, as for a caterpillar it was impossible to escape from the Petri dish/container. Survival was expressed as (living caterpillars)/(total number put on the leaf) and the total weight of the surviving caterpillars was measured in mg. For ANOVA analysis survival data were transformed as $\operatorname{arcsine}[\operatorname{sqrt}(\mathrm{x})]$ and caterpillar weight as $\log (\mathrm{x}+0.1)$.

Aphid data Survival was expressed as (living aphids)/(living + dead aphids); the total number of aphids could be less than 10 as some escaped from the cages, and cages with less than 4 aphids were discarded. The number of next generation nymphs was divided by the estimated average number of living aphids present, calculated as $(2 *$ living aphids + dead aphids)/2. For ANOVA analysis these data were transformed to obtain a more or less constant residual variance: survival as $\operatorname{arcsine}[\operatorname{sqrt}(\mathrm{x})]$ and number of next generation nymphs as $\log (\mathrm{x}+0.1)$.

For all experiments ANOVA analyses were performed with 15 accessions as treatments and 5 or 7 blocks, with corresponding degrees of freedom: 14 for the accessions, 4 or 6 for the blocks and 56 or 84 for the residuals (corrected for missing values as needed). Significance of differences of accession means was 
tested using an LSD test $(\mathrm{P}<0.05)$ on the transformed scales.

Correlations among the accession means (of the transformed data) of the different insect resistance parameters were calculated using the Pearson's correlation coefficient.

Metabolite data Non-detects were randomized between 45 and $55 \%$ of the local noise calculated by the Metalign software (at a signal to noise ratio of 3), resulting in values between 400 and 500 in the data matrix. Data were $\log 10$ transformed before carrying out t-tests (in Excel), or Principal Component Analysis (PCA) and Hierarchical Cluster Analysis (HCA), both using GeneMaths XT (Applied Maths, Belgium). A false discovery rate (FDR) correction was applied to correct for multiple comparisons. The corresponding q-values were calculated according to (Benjamini and Hochberg 1995). Before PCA and HCA the logtransformed data were normalized across samples prior to analysis. Cluster analysis was carried out using Pearson correlation and Unweighted Pair Group Method with Arithmetic Mean (UPGMA) clustering.

\section{Results}

Evaluation of the accessions for resistance to insects

Based on available information on genetic relationships, resistance to $B$. tabaci, presence/absence of trichome type IV and acyl sugar content among 12 accessions of the Lycopersicon group of Solanum, section Lycopersicon together with 3 reference accessions (Firdaus et al. 2012) were selected for further evaluation (Supplementary Table 1) using Trialeurodes vaporariorum, Frankliniella occidentalis, Spodoptera exigua and Myzus persicae. Evaluation data of the 15 accessions are presented in a condensed form in Fig. 1, whereas the full data set can be found in the Supplementary Tables 2-5). The significance of the accession effect was $<0.001$ in all experiments.

For the evaluation with the whitefly $T$. vaporariorum, two parameters (i.e. adult survival and oviposition) were used, which were highly correlated $(\mathrm{R}=0.96$, Table 1). On the $S$. pennellii accession LA716 and $S$. galapagense accession LA1401 all whiteflies died and only very few eggs were deposited (Supplementary Table 2). All S. cheesmaniae and most S. pimpinellifolium accessions were highly susceptible and almost all whiteflies survived on them.

Resistance to the thrips $F$. occidentalis was measured in a detached leaf assay where thrips survival after 2, 3 and 4 days was used as parameter. Analysis showed that differences among accessions were not significant after 2 days (ANOVA P $=0.23$ ), but were significant after 3 (ANOVA $\mathrm{P}=0.004$ ) and 4 (ANOVA $\mathrm{P}<0.001$ ) days (Add. Table 3 ). Thrips survival on days 3 and 4 were highly correlated $(\mathrm{R}=0.88$, Table 1$)$. After four days, all thrips were dead on the resistant accessions. Again the accession LA716 and LA1401 were among the most resistant, together with $S$. galapagense accession LA438, $S$. pimpinellifolium accession LA1580, and the $S$. habrochaites accessions (PI134418, LA1777) (Fig. 1).

Resistance to the caterpillar S. exigua was measured as larval survival and weight after 15 days in a detached leaf assay (Add. Table 4). Both parameters were highly correlated $(\mathrm{R}=0.93$, Table 1$)$. Figure 1 shows that all $S$. cheesmaniae and $S$. galapagense accessions were among the most resistant genotypes, on which only few caterpillars survived. In contrast, all $S$. pimpinellifolium accessions and cv. Moneymaker were susceptible to $S$. exigua.

For evaluation of resistance to the aphid $M$. persicae, both survival and the number of offspring produced were used as parameters. Results are shown in Supplementary Table 5. Both parameters appeared significantly correlated $(\mathrm{R}=0.83)$. The $S$. galapagense accessions were among the most resistant, together with $S$. pennellii LA716, both S. habrochaites accessions (LA1777, PI134418) and the S. pimpinellifolium accession LA1580, whereas the S. cheesmaniae accessions were among the most susceptible (Fig. 1).

Table 1 shows that the correlations between whitefly survival, thrips survival and aphid survival were high, whereas the correlations of these traits with caterpillar survival were low.

Metabolite profiles

Differences in metabolite profiles between leaves of the Lycopersicon group accessions were analyzed using an LCMS-based, essentially untargeted metabolomics approach (De Vos et al. 2007). The extraction of leaves with $75 \%$ methanol (acidified with $0.1 \%$ 


$\begin{array}{llllllll}\text { Whitefly } & & \text { Thrips } & & \text { Caterpillar } & & \text { Aphid } & \\ \text { LA716 } & \text { a } & \text { LA716 } & \text { a } & \text { LA746 } & \text { a } & \text { LA438 } & \text { a } \\ \text { LA1401 } & \text { ab } & \text { LA1401 } & \text { ab } & \text { LA1408 } & \text { a } & \text { LA1401 } & \text { a } \\ \text { LA1777 } & \text { bc } & \text { LA438 } & \text { abc } & \text { LA1777 } & \text { a } & \text { LA716 } & \text { a } \\ \text { PI134418 } & \text { c } & \text { LA1580 } & \text { abcd LA438 } & \text { a } & \text { LA1777 } & \text { a } \\ \text { LA438 } & \text { d } & \text { PI134418 } & \text { abcd LA1401 } & \text { a } & \text { PI134418 } & \text { a } \\ \text { LA1584 } & \text { de } & \text { LA1777 } & \text { abcd PI134418 } & \text { a } & \text { LA1584 } & \text { a } \\ \text { LA1408 } & \text { de } & \text { LA1408 } & \text { bcd } & \text { LA1139 } & \text { a } & \text { LA1408 } & \text { a } \\ \text { LA1580 } & \text { ef } & \text { LA746 } & \text { bcd } & \text { LA521 } & \text { a } & \text { LA1580 } & \text { b } \\ \text { CV. MM } & \text { ef } & \text { LA1139 } & \text { bcd } & \text { LA716 } & \text { a } & \text { LA1547 } & \text { bc } \\ \text { LA1596 } & \text { ef } & \text { LA521 } & \text { bcd } & \text { LA1547 } & \text { b } & \text { LA746 } & \text { bc } \\ \text { LA1139 } & \text { ef } & \text { LA1596 } & \text { cd } & \text { cV. MM } & \text { bc } & \text { cV. MM } & \text { bcd } \\ \text { LA746 } & \text { ef } & \text { LA1547 } & \text { cde } & \text { LA1584 } & \text { b } & \text { LA1596 } & \text { cd } \\ \text { LA1547 } & \text { ef } & \text { LA1584 } & \text { de } & \text { CGN14498 } & \text { d } & \text { LA1139 } & \text { de } \\ \text { LA521 } & \text { f } & \text { cV. MM } & \text { e } & \text { LA1580 } & \text { cd } & \text { CGN14498 } & \text { e } \\ \text { CGN14498 } & \text { f } & \text { CGN14498 } & \text { e } & \text { LA1596 } & \text { d } & \text { LA521 } & \text { e }\end{array}$

Legend:

S. habrochaites
S. cheesmaniae
S. galapagense
CV. Moneymaker
S. pimpinellifolium
S. pennellii

Fig. 1 Evaluation of accessions for resistance to four insect species. Shown is the relative position of an accession in the evaluation based on the survival data for the whitefly Trialeurodes vaporariorum, thrips Frankliniella occidentalis (after 4 days), caterpillar Spodoptera exigua (after 4 days) and the aphid Myzus persicae (after 12 days). At the top of each column is the accession with the lowest survival (which was

formic acid), analysis of the crude extracts by high mass resolution LCMS (Supplementary Fig. 1) followed by unbiased data processing resulted in a total of 2565 putative compounds, including secondary metabolites such as alkaloids, flavonoids, phenylpropanoids, acyl sugars, etcetera, detected among the accessions evaluated (Supplementary Table 6). The PCA plot based on the metabolite profiles (Fig. 2) shows a separation of plants into 3 distinct clusters: one for the $S$. galapagense accessions, one for the $S$. cheesmaniae accessions, and one for $S$. pimpinellifolium accessions plus cv. Moneymaker. Biological replicates per accession clearly group together, thereby validating the untargeted metabolomics approach applied as a reproducible, comprehensive phytochemical phenotyping tool enabling the identification of genetic effects on the plant metabolome zero on the most resistant accession in each evaluation) and at the bottom the highest (which was $100 \%$ in all evaluations, except thrips). If no letters behind the accession name are in common, mean values differ significantly based on the LSD test $(\mathrm{P}<0.05)$ within each evaluation. Aggregated data are based on Supplementary Tables 2-5. Different colors indicate different species. (Color figure online)

(see also Supplementary Fig. 1 which shows the HCA dendrogram that was constructed based on these metabolites). Within $S$. pimpinellifolium there is a division between, on the one hand, LA1580 and LA1584 and, on the other hand, LA1547, LA1596 and CGN14498 together with tomato cv Moneymaker.

Based on their specific accurate mass and retention times in the LCMS profiles, we were able to putatively annotate 138 different acyl sugar structures of which 41 were further characterized using MSMS and MS3 fragmentation (Supplementary Fig. 2). The different tomato species appear to produce different sets of acyl sugars (Fig. 3). Unique acyl sugars, i.e. acyl sugar structures detectable in only a single accession or species, were found for all species except cv. Moneymaker and the $S$. cheesmaniae accessions. Acylated forms of glucose, i.e. acyl glucoses, were detected not 
Table 1 Correlation coefficients among the insect resistance parameters

\begin{tabular}{|c|c|c|c|c|c|c|c|c|}
\hline & 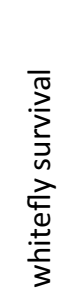 & 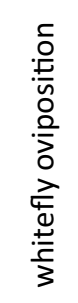 & 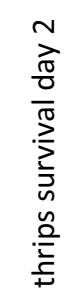 & 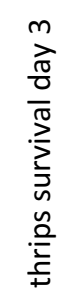 & 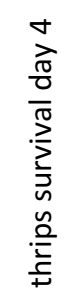 & 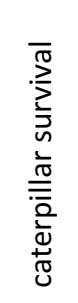 & 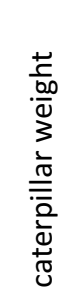 & $\begin{array}{l}\frac{\pi}{2} \\
\frac{2}{2} \\
\frac{1}{3} \\
\frac{0}{2} \\
\frac{0}{2} \\
\frac{0}{0}\end{array}$ \\
\hline whitefly oviposition & 0.96 & & & & & & & \\
\hline thrips survival day 2 & 0.59 & 0.63 & & & & & & \\
\hline thrips survival day 3 & 0.74 & 0.64 & 0.60 & & & & & \\
\hline thrips survival day 4 & 0.73 & 0.59 & 0.44 & 0.87 & & & & \\
\hline caterpillar survival & 0.49 & 0.41 & 0.19 & 0.43 & 0.62 & & & \\
\hline caterpillar weight & 0.46 & 0.40 & 0.21 & 0.41 & 0.58 & 0.99 & & \\
\hline aphid survival & 0.80 & 0.75 & 0.42 & 0.62 & 0.61 & 0.49 & 0.42 & \\
\hline aphid nymphs & 0.69 & 0.69 & 0.36 & 0.46 & 0.42 & 0.21 & 0.15 & 0.89 \\
\hline
\end{tabular}

Correlations were calculated using the transformed data for the different parameters for the 15 accessions. Correlations in orange, green and yellow are significantly different from 0 at $\mathrm{P}<0.001, \mathrm{P}<0.01$ and $\mathrm{P}<0.05$ respectively

Fig. 2 PCA analysis of the accessions based on all 2565 metabolites detected by LCMS. Accessions were analyzed in 2 independent biological replicates.

Yellow spheres $=S$. cheesmaniae samples, red $=S$. galapagense, green $=S$.

pimpinellifolium, and pink $=\mathrm{cv}$. Moneymaker. Explained variances are $25.5,19.7$ and $8.3 \%$ for the $\mathrm{x}, \mathrm{y}, \mathrm{z}$-axis, respectively. (Color figure online)

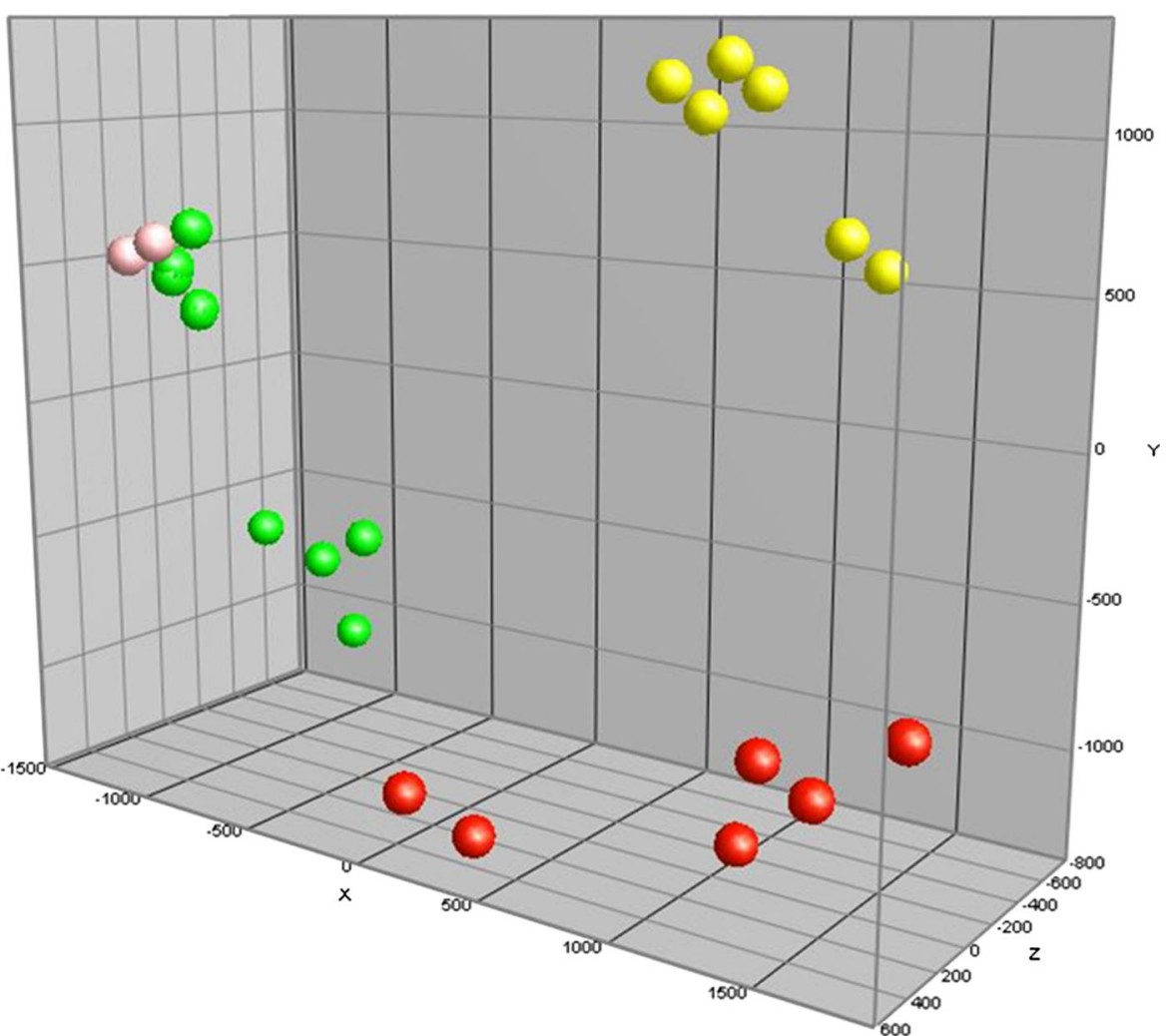


Fig. 3 UPGMA analysis based on 138 acyl sugars detected in the 15 Solanum accessions. Accession number is indicated at the bottom. Species to which an accession belongs is indicated by a colored square: light brown $=S$. pennellii, dark brown $=S$. habrochaites, pink $=S$. lycopersicum, green $=S$. pimpinellifolium, yellow $=S$. cheesmaniae, red $=S$. galapagense . Dendrograms on the right side (relation among acyl sugars) and top (relation among accessions) are based on Pearson correlation coefficients. The heat map is based on all acyl sugars annotated in Supplementary Table 7). Heat map color key: red high $(+4)$, green low $(-4)$ relative abundance of the compound. (Color figure online)

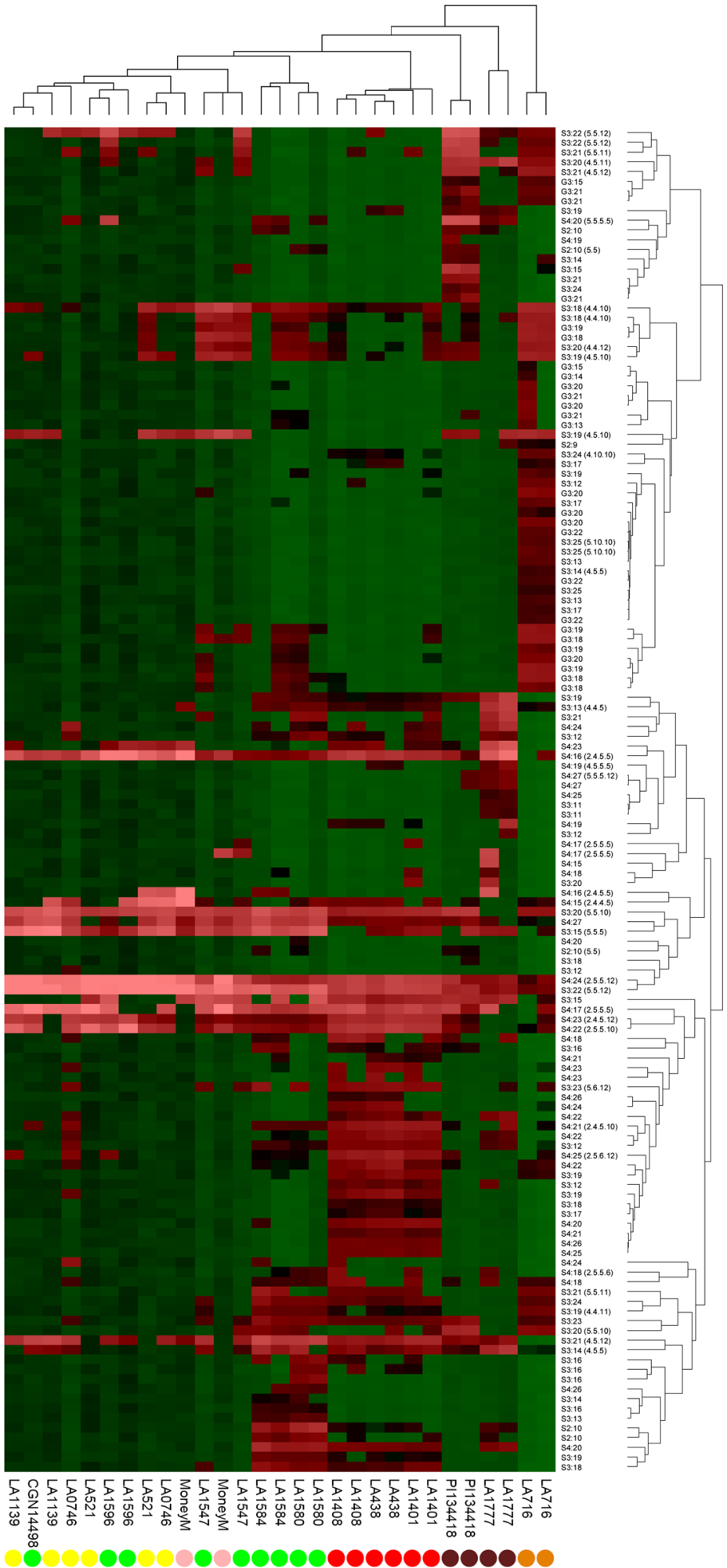

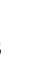


only in S. pennellii, conform earlier reports (Schilmiller et al. 2016) but also in some lines of $S$. pimpinellifolium and $S$. galapagense, although most of these acyl glucoses were present at relatively low levels as compared to $S$. pennellii. Both accessions of S. habrochaites accumulated a unique set of acyl sucroses. Likewise, the $S$. pimpinellifolium accessions LA1580 and LA1584 produced unique acyl sucroses, among which were 2 isomers of S2:10, i.e. sucrose acylated with only 2 acyl groups with a total length of 10 carbons. Solanum galapagense produced several unique S3 and S4 acyl sucroses (Supplementary Table 7).

The genetically closely-related species $S$. galapagense and $S$. cheesmaniae are clearly different at their metabolite level, as shown in Supplementary Fig. 3. Between the two species 260 metabolites significantly differed in their relative abundance (FDR $=0.01$ ), i.e. $10 \%$ of all metabolites detected. Of these differential metabolites 76 showed a $S$. galapagense/S. cheesmaniae $(\mathrm{SG} / \mathrm{SC}$ ) ratio $<0.5$ and 175 a SG/SC ratio $>2$. Sixty-four metabolites were at least 100 times more abundant in $S$. galapagense, while 9 were 100 times more abundant in $S$. cheesmaniae (Supplementary Table 6). Out of the 5 metabolites with the highest SG/ $\mathrm{SC}$ ratio, 4 represented different methylated forms of myricetin, a flavonol-type of flavonoid. These metabolites were all absent, i.e. below the detection limit, in $S$. cheesmaniae. Between S. galapagense and S. cheesmaniae accessions there were 39 differentiating acyl sugars, all of them with a sucrose backbone; 23 of these were at least 100 times more abundant in $S$. galapagense than in S. cheesmaniae (Supplementary Table 7).

\section{Discussion}

Insect resistance in the Lycopersicon group of Solanum section Lycopersicon

There are large and highly significant differences in insect resistance among accessions from the Lycopersicon group. When evaluating the sap sucking insects (whitefly, aphid) and the cell content feeder (thrips), the accessions from $S$. galapagense were among the most resistant, together with the reference accessions from S. pennellii and S. habrochaites which previously have been shown to be highly resistant to insects (Kennedy 2003). Within S. pimpinellifolium, resistance to some pest insects is found, i.e. thrips resistance in accession LA1580 and aphid resistance in LA1584. The other accessions of S. pimpinellifolium were very susceptible, even more susceptible than cv. Moneymaker. Accessions of $S$. cheesmaniae are as susceptible to sap sucking insects as cv. Moneymaker. The results obtained for $S$. galapagense and $S$. cheesmaniae accessions with regard to their contrasting resistance to the whitefly $T$. vaporariorum, as shown in the present study, are very similar to those previously reported for B. tabaci (Lucatti et al. 2013; Rakha et al. 2017b), suggesting a similar mechanism governing resistance against both whitefly species. The same mechanism may also account for resistance against thrips and aphids, as the data for adult survival of whitefly, thrips and the aphids are highly correlated. All insect resistant accessions possess glandular trichomes type I and IV on their leaves, the vast majority of them being type IV, strongly suggesting that these trichomes are essential for plant resistance, as was shown previously (Firdaus et al. 2012; Lucatti et al. 2013). Considering the plant tissues on which whiteflies, aphids and thrips feed, it is unlikely that these insects will ingest phytochemicals present in the glandular trichomes. However, their bodies and wings may get into direct contact with compounds actively secreted by, or released from, the type I and/or IV trichomes. It has been suggested that the sticky contents released by these trichomes can immobilize the insects on the leaf surface, ultimately leading to the death of the insect (Rakha et al. 2017a; Schilmiller et al. 2008). Indeed, we observed dead insects "glued" onto the leaves of trichome type I and IV containing accessions in our clip cage assays.

Interestingly, the results obtained for the chewing $S$. exigua larvae were quite different from those obtained for the sap sucking and cell content feeding pest insects. The $S$. cheesmaniae accessions show a $S$. exigua resistance level similar to that of $S$. galapagense accessions, suggesting that resistance against $S$. exigua is likely based on a different mechanism (or compounds) than the resistance against the sap suckers. Larvae of $S$. exigua consume whole leaves and are thus ingesting and exposed to all compounds present in the various leaf cells, including the trichomes, while sap suckers are only ingesting phloem sap. All S. pimpinellifolium accessions were very susceptible to $S$. exigua, suggesting that the 
hypothetical resistance related compound(s) is absent in this species. However, many metabolites were found to be differential between the, relatively few, $S$. pimpinellifolium and $S$. cheesmaniae accessions investigated in the present study (Supplementary Tables 1 and 7). To get more insight into the key compound(s) involved in S. exigua resistance it is necessary to compare larger numbers of different accessions from both $S$. pimpinellifolium and $S$. lycopersicum, on the one hand, and both $S$. galapagense and $S$. cheesmaniae, on the other hand. Alternatively, segregating populations of $S$. galapagense $\times$ S. lycopersicum (or S. pimpinellifolium) crosses may be used.

Leaf metabolites and their relation to the Wf1-QTL

Accessions from the three wild species $S$. pimpinellifolium, $S$. galapagense and $S$. cheesmaniae form distinct groups at their metabolite level. Within the $S$. pimpinellifolium accessions, there appear to be two distinct groups based on differences in their metabolite profiles: one containing the accessions LA1580 and LA1584 and the other containing LA1547, LA1596 and CGN14498. This division within S. pimpinellifolium accessions is related to the presence/absence of type IV trichomes, which are absent in the latter three accessions.

Despite the small differences at their genome level (Lucatti et al. 2013), large differences exist in the metabolomes between $S$. galapagense and $S$. cheesmaniae accessions. The abundances of 260 metabolites were significantly different between the two species, which is about $10 \%$ of the total number of detected metabolites. Such large differences may be due to common biochemical processes, clustering of genes involved in the synthesis of metabolites or regulatory mechanisms affecting the presence/absence of trichomes type I and IV. In a previous paper, using a $S$. galapagense $\times$ S. lycopersicum $\mathrm{F} 2$ population, we have shown that both whitefly resistance and the production of type IV trichomes is controlled by a major QTL, designated $W f-1$, located on the end of Chromosome 2. In addition, resistant and susceptible plants of this F2 population showed large differences in the composition and content of acyl sugars, relate to the presence/absence of the type IV trichomes (Firdaus et al. 2013). The $S$. galapagense and S. cheesmaniae plants analyzed here show a similar contrast in whitefly resistance related to the presence or absence of trichome type IV and acyl sugar content. Therefore we speculate that the same $W f-1$ QTL, identified by the $S$. galapagense $\times S$. lycopersicum crossing, is also underlying the observed resistance-related differences between $S$. galapagense and $S$. cheesmaniae, and evaluated this region for candidate genes related to the production of differentially expressed metabolites and/or the production of the glandular trichome itself. Among the compounds with the highest $S$. galapagense/S. cheesmaniae (SG/SC) ratios, different $O$ methylated forms of myricetin and one of quercetin, both belonging to the flavonol-type of flavonoids, were within the top 6 of differentially accumulated compounds (Supplementary Table 6). Myricetin methyl transferases have been identified on chromosome VI of S. habrochaites, and transcripts of these genes were shown to be present in glandular trichomes (Kim et al. 2014; Schmidt et al. 2011). In the Wf-I QTL region of the reference sequence Heinz 1706, between position SL2.50ch02: 53768859 and the end of the chromosome, there are four candidate genes annotated as caffeoyl-CoA $O$-methyltransferases (IPR002935 $O$-methyltransferase) which may be involved in the methylation of these flavonol-type of phenolic compounds (Supplementary Table 8)). We hypothesize that at least one of these $O$-methyltransferases can act as a flavonol $O$-methyl transferase leading to the accumulation of the $O$-methylated myricetin and quercetin. Although several flavonoids (Morant et al. 2003; Treutter 2005) have been implicated in resistance against pathogens and herbivores, it remains to be established whether these methylated forms of myricetin and quercetin are directly involved in whitefly resistance.

Between the different accessions carrying glandular trichomes type IV there are also large differences in the acyl sugar composition, in which each plant species accumulates a specific set of acyl sugars (Fig. 3). Several acyl glucoses are exclusively produced by $S$. pennellii, as was shown before (Blauth et al. 1998; McDowell et al. 2011). Other species mainly produce acyl sucroses. Relative high levels of specific acyl sugars are also found in both $S$. habrochaites, the S. pimpinellifolium with type IV trichomes and the $S$. galapagense accessions. The fact that species-specific acyl sugars are observed may be related to the presence or absence of specific acyl transferases (Fan et al. 2016) or CXE carboxylesterase 
(Schilmiller et al. 2016), which are involved in the production and modification of acyl sugars, respectively. For 39 different acyl sugar structures, i.e. almost $30 \%$ of the total acyl sugar structures identified, the relative abundance differed significantly between $S$. galapagense and $S$. cheesmaniae accessions with all of them being higher in S. galapagense. With regard to the production of these acyl sugars, the Wf1-QTL region contains one putative candidate gene harboring the IPR003480 domain encoding acyl transferases (Fan et al. 2016). This candidate gene is annotated as a $N$-hydroxycinnamoyl/benzoyltransferase 3 , and it remains to be established whether the corresponding protein is able to esterify glucose and/ or sucrose molecules with aliphatic carbon chains to produce acyl sugars. In the same Wfl-QTL region there is also one gene that encodes a putative CXE carboxylesterase, which may be involved in modification of acyl sugars (Schilmiller et al. 2016). It should be noted that the candidate genes mentioned above may also show up if they are closely linked to the actual causal gene.

In principle, any or all of the compounds that are significantly different between $S$. galapagense and $S$. cheesmaniae may be responsible for the differential resistance against whiteflies, aphids and thrips. For several compounds mentioned above, i.e. acyl sugars, methylated flavonols, it has been shown that these are specifically produced or accumulating in the glandular trichomes (Glas et al. 2012). The fact that a large number of metabolites is differentially expressed between $S$. galapagense and $S$. cheesmaniae suggests a common biochemical process or regulatory mechanism being responsible for the observed differences in the metabolome. Such a key process could be the formation of the type IV trichomes itself, thus regulating the production and accumulation of a large group of defense compounds. It may even be hypothesized that only the formation of the head cell is key for the accumulation of these trichome-specific metabolites. Both head cell formation and the accumulation of its specific compounds could be under control of one or more transcription factors, similar to the situation of trichome formation in Arabidopsis thaliana (Pattanaik et al. 2014; Zhou et al. 2014). Obviously, there will be other genes required for the production of these metabolites as well, so there may be additional QTLs at other places in the genome for several of the metabolites. The resistance controlling
Wf1-QTL region contains several genes encoding transcription factors (see Additional Table S8). We hypothesize that at least one of these transcription factors within this Wf1-QTL region is involved in trichome or head cell formation, thereby regulating the accumulation of metabolites conferring insect resistance. Clearly, further research is needed to shed light on the possible involvement of candidate genes in this Wf1-QTL region.

Sources of resistance that may be used to increase insect resistance in cultivated tomato

In greenhouse cultivation most pest insects can be controlled biologically by natural enemies, e.g. predators and parasitoids that are released in the greenhouse (Hoddle et al. 1998; Van Lenteren 2000). However, in the open field the effect of biological control is not sufficient to remain below the damage threshold levels, and thus insecticides are used frequently. Yet, some insect species are difficult to control with insecticides and they develop resistance to the insecticides very quickly (Alyokhin et al. 2015). In addition, several of the insecticides are hazardous to the environment, which also urges for alternative solutions to reduce or prevent damage caused by insects. The best strategy is to prevent the insect problems from occurring by using resistant varieties. Tomato wild relatives constitute a large resource for all kinds of traits, which may be used to improve cultivated tomato. However, attempts to use wild relatives as sources of resistance have until now not resulted in insect resistant tomatoes (Lawson et al. 1997; Leckie et al. 2012). This is most likely due to the complex genetics of the resistance mechanism within these species, involving several QTLs (van den Oevervan den Elsen et al. 2016). Also, crossings with wild species are often difficult and linkage drag is not easily removed by back-crossing. The recently identified sources of insect resistance in the Lycopersicon group of Solanum section Lycopersicon may solve this problem to a large extent, as species within this group are closely related to the cultivated tomato and easier to cross, and also may carry less unfavourable alleles. Based on the results presented here and by others (Rakha et al. 2017a, b), especially the S. galapagense accessions are very promising for future breeding efforts towards improved, insect resistant tomato varieties. The resistance in $S$. galapagense is a broad 
spectrum type of resistance, largely based on the presence of glandular trichomes and its phytochemical content. The presence-absence of type IV trichomes was mapped to the Wf1-QTL on the end of chromosome II of S. galapagense, which is also responsible for B. tabaci resistance (Firdaus et al. 2013). Therefore, it is likely that this specific QTL also governs the resistance towards the other insects. Such a broad spectrum resistance is especially useful in open field cultivation systems, where a whole suite of insects can freely fly and infest the crop.

\section{Conclusions}

A broad spectrum resistance towards insects has been identified in close relatives of the cultivated tomato. Trichome type IV containing species from the Lycopersicon group of Solanum section Lycopersicon show resistance to a range of pest insects. Especially accessions of $S$. galapagense are highly resistant. Comparison of the metabolite content of $S$. galapagense to the genetically very similar $S$. cheesmaniae, which does not contain type IV trichomes, shows large differences in the levels of both acyl sugars and several O-methylated forms of the flavonol myricetin. The Wf1-QTL region previously identified by us contains several structural and transcription factor genes that may be responsible for the observed differences in insect resistance and metabolites between the two species. The broad spectrum insect resistance of $S$. galapagense holds great promises for transfer into cultivated tomato and will be especially useful in open field cultivation systems where a whole suite of insects can attack the crop.

Acknowledgements The authors would like to thank Greet Steenhuis-Broers for excellent technical assistance and advice on experiments with the insects, and Bert Schipper for operating the LCMS. We also thank Dr. Joop van Loon for critically reading the manuscript and valuable suggestions for improvement. This project was funded by a Grant (TKI 1310-071) from the Ministry of Economic Affairs of The Netherlands and the breeding companies Axia Vegetable Seeds B.V., The Netherlands, Enza Zaden Research \& Development B.V., The Netherlands, Rijk Zwaan Zaadteelt en Zaadhandel B.V., The Netherlands and East-West Seed Company, Inc., The Philippines.

Open Access This article is distributed under the terms of the Creative Commons Attribution 4.0 International License (http:// creativecommons.org/licenses/by/4.0/), which permits unrestricted use, distribution, and reproduction in any medium, provided you give appropriate credit to the original author(s) and the source, provide a link to the Creative Commons license, and indicate if changes were made.

\section{References}

Agrawal AA (2011) Current trends in the evolutionary ecology of plant defence. Funct Ecol 25:420-432. https://doi.org/ 10.1111/j.1365-2435.2010.01796.x

Alba JM, Montserrat M, Fernandez-Munoz R (2009) Resistance to the two-spotted spider mite (Tetranychus urticae) by acylsucroses of wild tomato (Solanum pimpinellifolium) trichomes studied in a recombinant inbred line population. Exp Appl Acarol 47:35-47. https://doi.org/10.1007/ s10493-008-9192-4

Alyokhin A et al (2015) The Red Queen in a potato field: integrated pest management versus chemical dependency in Colorado potato beetle control. Pest Manag Sci 71:343-356. https://doi.org/10.1002/ps.3826

Benjamini Y, Hochberg Y (1995) Controlling the false discovery rate-a practical and powerful approach to multiple testing. J R Stat Soc Ser B (Methodol) 57:289-300

Blauth SL, Churchill GA, Mutschler MA (1998) Identification of quantitative trait loci associated with acylsugar accumulation using intraspecific populations of the wild tomato, Lycopersicon pennellii. Theor Appl Genet 96:458-467. https://doi.org/10.1007/s001220050762

Broekgaarden C, Snoeren TA, Dicke M, Vosman B (2011) Exploiting natural variation to identify insect-resistance genes. Plant Biotechnol J 9:819-825. https://doi.org/10. 1111/j.1467-7652.2011.00635.x

Darwin SC, Knapp S, Peralta IE (2003) Taxonomy of tomatoes in the Galápagos Islands: native and introduced species of Solanum section Lycopersicon (Solanaceae). Syst Biodivers 1:29-53. https://doi.org/10.1017/s1477200003001026

De Vos RCH, Moco S, Lommen A, Keurentjes JJ, Bino RJ, Hall RD (2007) Untargeted large-scale plant metabolomics using liquid chromatography coupled to mass spectrometry. Nat Protoc 2:778-791. https://doi.org/10.1038/nprot. 2007.95

Fan P et al (2016) In vitro reconstruction and analysis of evolutionary variation of the tomato acylsucrose metabolic network. Proc Natl Acad Sci USA 113:E239-E248. https:// doi.org/10.1073/pnas.1517930113

Firdaus S, van Heusden AW, Hidayati N, Supena EDJ, Visser RGF, Vosman B (2012) Resistance to Bemisia tabaci in tomato wild relatives. Euphytica 187:31-45. https://doi. org/10.1007/s10681-012-0704-2

Firdaus S et al (2013) Identification and QTL mapping of whitefly resistance components in Solanum galapagense. Theor Appl Genet 126:1487-1501. https://doi.org/10. 1007/s00122-013-2067-z

Glas JJ, Schimmel BC, Alba JM, Escobar-Bravo R, Schuurink RC, Kant MR (2012) Plant glandular trichomes as targets for breeding or engineering of resistance to herbivores. Int J Mol Sci 13:17077-17103. https://doi.org/10.3390/ ijms 131217077 
Hoddle MS, Van Driesche RG, Sanderson JP (1998) Biology and use of the whitefly parasitoid Encarsia formosa. Annu Rev Entomol 43:645-669. https://doi.org/10.1146/ annurev.ento.43.1.645

Kennedy GG (2003) Tomato, pests, parasitoids, and predators: tritrophic interactions involving the genus Lycopersicon. Annu Rev Entomol 48:51-72. https://doi.org/10.1146/ annurev.ento.48.091801.112733

Kim J et al (2014) Analysis of natural and induced variation in tomato glandular trichome flavonoids identifies a gene not present in the reference genome. Plant Cell 26:3272-3285. https://doi.org/10.1105/tpc.114.129460

Lawson DM, Lunde CF, Mutschler MA (1997) Marker-assisted transfer of acylsugar-mediated pest resistance from the wild tomato, Lycopersicon pennellii, to the cultivated tomato, Lycopersicon esculentum. Mol Breed 3:307-317. https://doi.org/10.1023/A:1009677412902

Leckie BM, De Jong DM, Mutschler MA (2012) Quantitative trait loci increasing acylsugars in tomato breeding lines and their impacts on silverleaf whiteflies. Mol Breed 30:1621-1634. https://doi.org/10.1007/s11032-012-9746-3

Lommen A (2009) MetAlign: interface-driven, versatile metabolomics tool for hyphenated full-scan mass spectrometry data preprocessing. Anal Chem 81:3079-3086. https://doi. org/10.1021/ac900036d

Lommen A, Gerssen A, Oosterink JE, Kools HJ, Ruiz-Aracama A, Peters RJ, Mol HG (2011) Ultra-fast searching assists in evaluating sub-ppm mass accuracy enhancement in U-HPLC/orbitrap MS data. Metabolomics 7:15-24. https:// doi.org/10.1007/s11306-010-0230-y

Lucatti AF, van Heusden AW, de Vos RC, Visser RG, Vosman B (2013) Differences in insect resistance between tomato species endemic to the Galapagos Islands. BMC Evol Biol 13:175. https://doi.org/10.1186/1471-2148-13-175

Luu VT et al (2017) O-acyl sugars protect a wild tobacco from both native fungal pathogens and a specialist herbivore. Plant Physiol 174:370-386. https://doi.org/10.1104/pp.16. 01904

McDowell ET et al (2011) Comparative functional genomic analysis of Solanum glandular trichome types. Plant Physiol 155:524-539. https://doi.org/10.1104/pp.110. 167114

Moco S et al (2007) Tissue specialization at the metabolite level is perceived during the development of tomato fruit. J Exp Bot 58:4131-4146. https://doi.org/10.1093/jxb/erm271

Morant M, Bak S, Møller BL, Werck-Reichhart D (2003) Plant cytochromes P450: tools for pharmacology, plant protection and phytoremediation. Curr Opin Biotechnol 14:151-162. https://doi.org/10.1016/s0958-1669(03)00024-7

Muigai SG, Bassett MJ, Schuster DJ, Scott JW (2003) Greenhouse and field screening of wild Lycopersicon germplasm for resistance to the whitefly Bemisia argentifolii. Phytoparasitica 31:27-38

Pattanaik S, Patra B, Singh SK, Yuan L (2014) An overview of the gene regulatory network controlling trichome development in the model plant. Arabidopsis Front Plant Sci 5:259. https://doi.org/10.3389/fpls.2014.00259

Peralta IE, Spooner DM (2005) Morphological characterization and relationships of wild tomatoes (Solanum L. Section Lycopersicon). Monogr Syst Bot Mo Bot Gard 104:227-257
Peralta IE, Spooner DM, Knapp S (2008) Taxonomy of wild tomatoes and their relatives (Solanum sect. Lycopersicoides, sect. Juglandifolia, sect. Lycopersicon: Solanaceae). American Society of Plant Taxonomists, Ann Arbor

Rakha M, Bouba N, Ramasamy S, Regnard J-L, Hanson P (2017a) Evaluation of wild tomato accessions (Solanum spp.) for resistance to two-spotted spider mite (Tetranychus urticae Koch) based on trichome type and acylsugar content. Genet Resour Crop Evol 64:1011-1022. https://doi. org/10.1007/s10722-016-0421-0

Rakha M, Hanson P, Ramasamy S (2017b) Identification of resistance to Bemisia tabaci Genn. in closely related wild relatives of cultivated tomato based on trichome type analysis and choice and no-choice assays. Genet Resour Crop Evol 64:247-260. https://doi.org/10.1007/s10722015-0347-y

Rodriguez-Lopez MJ, Garzo E, Bonani JP, Fereres A, Fernandez-Munoz R, Moriones E (2011) Whitefly resistance traits derived from the wild tomato Solanum pimpinellifolium affect the preference and feeding behavior of Bemisia tabaci and reduce the spread of Tomato yellow leaf curl virus. Phytopathology 101:1191-1201. https://doi.org/10. 1094/PHYTO-01-11-0028

Roldan MVG et al (2014) Metabolomics reveals organ-specific metabolic rearrangements during early tomato seedling development. Metabolomics 10:958-974. https://doi.org/ 10.1007/s11306-014-0625-2

Schilmiller AL, Last RL, Pichersky E (2008) Harnessing plant trichome biochemistry for the production of useful compounds. Plant J 54:702-711. https://doi.org/10.1111/j. 1365-313X.2008.03432.x

Schilmiller AL, Charbonneau AL, Last RL (2012) Identification of a BAHD acetyltransferase that produces protective acyl sugars in tomato trichomes. Proc Natl Acad Sci USA 109:16377-16382. https://doi.org/10.1073/pnas.1207906109

Schilmiller AL, Moghe GD, Fan P, Ghosh B, Ning J, Jones AD, Last RL (2015) Functionally divergent alleles and duplicated Loci encoding an acyltransferase contribute to acylsugar metabolite diversity in Solanum trichomes. Plant Cell 27:1002-1017. https://doi.org/10.1105/tpc.15.00087

Schilmiller AL, Gilgallon K, Ghosh B, Jones AD, Last RL (2016) Acylsugar acylhydrolases: carboxylesterase-catalyzed hydrolysis of acylsugars in tomato trichomes. Plant Physiol 170:1331-1344. https://doi.org/10.1104/pp.15. 01348

Schmidt A, Li C, Shi F, Jones AD, Pichersky E (2011) Polymethylated myricetin in trichomes of the wild tomato species Solanum habrochaites and characterization of trichome-specific $3^{\prime} / 5^{\prime}$ - and 7/4'-myricetin $O$-methyltransferases. Plant Physiol 155:1999-2009. https://doi.org/10. 1104/pp.110.169961

Smith CM, Boyko EV (2007) The molecular bases of plant resistance and defense responses to aphid feeding: current status. Entomol Exp Appl 122:1-16. https://doi.org/10. 1111/j.1570-7458.2006.00503.x

Thompson JN (2005) Coevolution: the geographic mosaic of coevolutionary arms races. Curr Biol 15:R992-R994. https://doi.org/10.1016/j.cub.2005.11.046

Tikunov YM, Laptenok S, Hall RD, Bovy A, de Vos RC (2012) MSClust: a tool for unsupervised mass spectra extraction of chromatography-mass spectrometry ion-wise aligned 
data. Metabolomics 8:714-718. https://doi.org/10.1007/ s11306-011-0368-2

Treutter D (2005) Significance of flavonoids in plant resistance and enhancement of their biosynthesis. Plant Biol 7:581-591. https://doi.org/10.1055/s-2005-873009

van den Oever-van den Elsen F, Lucatti AF, van Heusden S, Broekgaarden C, Mumm R, Dicke M, Vosman B (2016) Quantitative resistance against Bemisia tabaci in Solanum pennellii: genetics and metabolomics. J Integr Plant Biol 58:397-412. https://doi.org/10.1111/jipb.12449 van der Hooft JJJ, Vervoort J, Bino RJ, de Vos RCH (2011) Spectral trees as a robust annotation tool in LC-MS based metabolomics. Metabolomics 8:691-703. https://doi.org/ 10.1007/s11306-011-0363-7

Van Lenteren JC (2000) A greenhouse without pesticides: fact or fantasy? Crop Prot 19:375-384

Zhou L, Zheng K, Wang X, Tian H, Wang X, Wang S (2014) Control of trichome formation in Arabidopsis by poplar single-repeat R3 MYB transcription factors. Front Plant Sci 5:262. https://doi.org/10.3389/fpls.2014.00262 